\title{
Unraveling Fire Suppression Sprays
}

\author{
ANDRE W. MARSHALL \\ Department of Fire Protection Engineering \\ University of Maryland \\ College Park, Maryland 20742 USA
}

\begin{abstract}
Spray generation represents a tremendously effective means of dispersing water for fire suppression applications. A variety of spray generation methods ranging from simple hose stream approaches to sophisticated water mist techniques have been developed and refined over the past century to address a diversity of fire protection challenges. Although the basic mechanisms for water based fire suppression are readily apparent, the underlying physics governing spray initiation (i.e. atomization) and the associated nozzle discharge characteristics are poorly understood. As a result, engineering analysis of these devices is riddled with empiricism from design conceptualization to performance evaluation. While computational design tools are becoming increasingly popular for a variety of fire protection applications, lack of knowledge regarding spray generation has limited the utility of computational tools for fire suppression problems. This paper describes a number of recent advancements in the analysis of fire suppression sprays. These experimental and analytical advancements have provided clarity in characterizing these complex sprays while creating critical pathways for the development of computational based approaches to support the design and analysis of water based fire suppression systems.
\end{abstract}

KEYWORDS: sprinklers, suppression, water sprays.

\section{INTRODUCTION}

A variety of fire suppression nozzles and systems have been developed to contend with a myriad of fire scenarios. Despite their variety, these nozzles and systems are all designed with the goal of suppressing the fire by strategically dispersing the water. Strategies vary from surface cooling by transient localized flooding (hose and monitor systems), surface cooling by distributed uniform surface wetting (sprinkler systems), to gas cooling and dilution by air wetting (mist systems). Although the basic suppression strategies are straightforward, describing the spray dispersion physics quantitatively is a challenging problem. This problem, among others, must be solved in order to predict suppression performance with any degree of fidelity.

The goal of this paper is to demonstrate how advanced measurement and analytical capabilities can be used to achieve detailed descriptions of fire suppression sprays. Recent research focused on sprinkler spray discharge characteristics (the initial spray) will be used to exemplify how sophisticated analytical and experimental techniques combine to provide insight into the complex physics responsible for generating fire suppression sprays. The uncertainty in defining the associated initial spray and related discharge characteristics makes analysis of spray dispersion and the associated fire suppression performance difficult from the very beginning. A significant first step in the analysis of fire suppression would be to develop predictive capability for the volume flux of water delivered to a target area or region. Accomplishing this goal would not only be scientifically noteworthy, but also practically useful as much of the fire suppression engineering practice and even regulation is based on critical volume fluxes of water. It is the author's hope that recent advancements in sprinkler spray research provided in this paper will demonstrate that complex sprays do yield themselves to quantitative treatment and that this progress will inspire similar developments in other fire suppression applications.

\section{Challenge}

The goal of characterizing the initial spray from fire suppression nozzles is ambitious because of the inherent complexity of real sprays. These nozzles rely on physical atomization mechanisms that are unstable and chaotic. Predicting the initial spray is a challenge for many industries and is not unique to fire suppression. However, fire suppression spray analysis is particularly challenging because the nozzle designs vary greatly, rely on different atomization mechanisms, and operate over a vast range of length and 
time scales. Despite their variety, water based suppression devices are designed to disperse water by generating a spatially distributed array of drops having stochastic local distributions of concentration, velocity, and drop size, which themselves have a complex spatial distribution even at initiation. The inherent complexity makes exact description of the initial spray difficult, notwithstanding the measurement challenges associated with the intractable multiphase breakup region, modeling challenges presented by the small scale free surface physics, and strong coupling with the dynamic fire environment. Despite these challenges, significant progress has been made to address this complexity for fire sprinkler sprays, which will be presented in this paper.

\section{Previous Studies}

There is no shortage of studies in the fire protection literature on water based fire suppression because of the importance of this topic. Many of the early studies focused on establishing criteria for fire suppression and identifying the important physical for a particular fire suppression technology such as Refs. [1-3] for sprinklers, Refs. [1,2,4] for hose streams, and Refs. [2,5-9] for water mist systems.

An important review of water-based suppression systems was conducted by Rasbash where he explores suppression performance and its dependence on fire and spray properties [1]. He distinguishes between flame and fuel cooling performance, while identifying the important properties of the fire source (e.g. phase/type, temperature, and burning rate) and important properties of the suppression spray (e.g. orientation, momentum, volume flux, and drop size). He evaluates suppression performance over a wide range of source/spray properties, explaining qualitative observations in terms of the important physical suppression mechanisms. Spray momentum and vaporization rate were quantified in certain tests and identified as being important properties for flame cooling, while critical surface volume fluxes were summarized and identified as important for fuel cooling. This review provided insight into the important suppression physics based on the limited data available, while highlighting the need for deeper understanding of the suppression process including pyrolysis during fuel wetting, extinction from spray vaporization, and kinematic interactions between the fire and the spray (dispersion).

The physical mechanisms driving suppression performance depend largely on the spray characteristics generated by the fire suppression device. As a result, a number of studies have focused on characterizing the sprays from fire suppression devices. Most notably, the review by Grant et al. covers a range of fire suppression sprays from water mist systems to hose streams [2]. The review performed by Grant et al. provide fire suppression spray details, the central topic of the current study, as well as an extensive discussion of fire suppression test data using a variety of nozzle types from water mist systems to hose streams. Typical discharge characteristics from these nozzles are discussed along with introductory material explaining how spray discharge characteristics are quantified and measured. While Rasbash reviewed the suppression for a variety of fuel sources, Grant et al. focus attention primarily on Class A (solid fuel) fires. Similar to Rasbash, they identify surface cooling, flame zone cooling, and oxygen displacement as the important suppression mechanisms and provides some fundamental qualitative discussion of observed suppression performance along with empirical analysis. From this excellent review, a number of specific insights were summarized. These insights were based on detailed presentation of the important spray nozzle classes, spray properties, and physical suppression mechanisms; however, the absence of a unifying fundamental framework for suppression analysis is clearly discernible from this review.

The current study aims to address the need for fundamental suppression research by advancing understanding of fire sprinkler sprays. Despite the relatively narrow focus of the current research on fire sprinklers, the advancements and approaches developed in this fire sprinkler research should demonstrate the possibilities and advantages of a high fidelity approach to fire suppression sprays in general. As sprinkler sprays will be the main focus of this paper, a brief literature review on this topic is provided in the following.

A number of earlier studies have laid the foundation for detailed treatment of the sprinkler spray. Dundas' early optical shadow-based measurements showed the effectiveness of this technique for large drop size sprays, although only a limited number of measurements were possible due to hardware and processing constraints [10]. Dundas' study also provided some information on the effect of geometry on the characteristic drop size, $d_{v 50}$, especially with regard to the orifice diameter, $D_{o}$. He was able to correlate his measurements and others using a correlation first proposed by Heskestad [11] 
where $W e=\rho_{l} U^{2} D_{o} / \sigma$ is the Weber number, $\rho_{l}$ is the liquid density, $U$ is the injection velocity, and $\sigma$ is the surface tension. Dundas found that the correlating coefficient varied from 1.74 to 3.21 depending on the sprinkler geometry. It should be noted that these early measurements provided only an overall drop size distribution without capturing spatial variation details of the sprinkler spray. Furthermore, these overall measured drop size distributions were based on local spatial measurements (or an average set of local spatial measurements), which strictly speaking produce a velocity bias. In the shadow images, lower velocity drops appear closer together and are thus measured more frequently. The spatial-based distribution does not necessarily agree with the flux-based drop size distribution, which corresponds to the drop size distribution actually delivered by the spray [12]. When velocity measurements are obtained simultaneously with drop size (as in later studies), this velocity bias can be removed to obtain flux-based drop size distributions. Yu [13] also used a system similar to that of Dundas and measured large orifice sprinklers with coefficients ranging from 2.33 to 4.48 , which is consistent with later measurements performed by Ren [14] who found the coefficient to be approximately 3.24 for pendent sprinkler with orifice diameters ranging from 5 to $18 \mathrm{~mm}$. Focusing on water distribution at the floor, Beyler performed extensive volume flux measurements in commercial sprinklers [15]. His volume flux distribution or 'patternation' results are extremely useful in quantifying the shadowing effect of frame arms on the spray distribution. In an experimental and analytical study, Prahl and Wendt evaluated sprinkler sprays beginning at the initial breakup responsible for spray generation and continuing to the dispersion process responsible for volume flux delivery [16]. They employed stability theory and novel experimental techniques to measure and analyze the critical wavelength leading to breakup in a laboratory sprinkler. They also employed a novel inviscid length scale to non-dimensionalize the location of volume flux measurements revealing drag effects and correlating data over a range of injection pressures.

More recently, Widmann measured discharge characteristics of residential sprinklers using a phase Doppler interferometer (PDI) [17]. Widmann obtained velocity and drop size information at a number of radial locations verifying the applicability of the PDI technique to sprinkler sprays. Sheppard performed a comprehensive set of experiments on 16 commercially available pendent and upright sprinklers $[18,19]$. Employing PDI techniques, he obtained local measurements of drop size at various azimuthal and elevation angles. Sheppard also applied PIV to measure drop velocity. The velocity and drop size data, presented in spherical coordinates with the sprinkler head at the center, showed significant spatial variation (with elevation angle). However, the drops near the sprinkler discharge $(\sim 0.2 \mathrm{~m}$ from the injection point) appeared to move radially outward with a velocity lower than $60 \%$ of the Bernoulli injection velocity. Putorti applied a particle tracking velocimetry and imaging (PTVI) technique on laboratory sprinkler configurations to measure drop size, velocity, trajectory, and mass flux [20]. His measurement approach was similar to the shadowgraphy method used in the current study; however, his drops were imaged using laser induced fluorescence. Most recently, Blum [21], Do, [22], and Ren [23] have performed a series of spray characterization studies on laboratory and actual fire sprinkler configurations which are summarized in the current paper. These studies have evaluated the topology of the initial spray revealing different spray characteristics in the vicinity of the tines and slots [24-26]. These initial spray features were characterized and analyzed with unprecedented detail. They provided unique insight into sprinkler geometry effects and three dimensional initial spray structure for spray analysis and modeling. Recognizing the increasing popularity of CFD modeling in fire protection engineering, this research sought not only to deepen understanding of sprinkler sprays, but also to provide this information in a framework that is suitable for CFD integration. To this end spray, measurements and analyses have been conducted in these studies to unravel the sprinkler spray.

\section{APPROACH}

\section{Perspective}

A typical pendent sprinkler configuration is provided in Fig. 1 highlighting the important geometric features of these devices. It should be noted that the sprinkler design is driven by a number of factors including link integration (i.e. activation), durability, and cost. It follows then that the overall performance 
of these devices is not determined solely by their spray generation capabilities. Nevertheless, spray generation is central to suppression performance and is discussed without qualification in the current study.
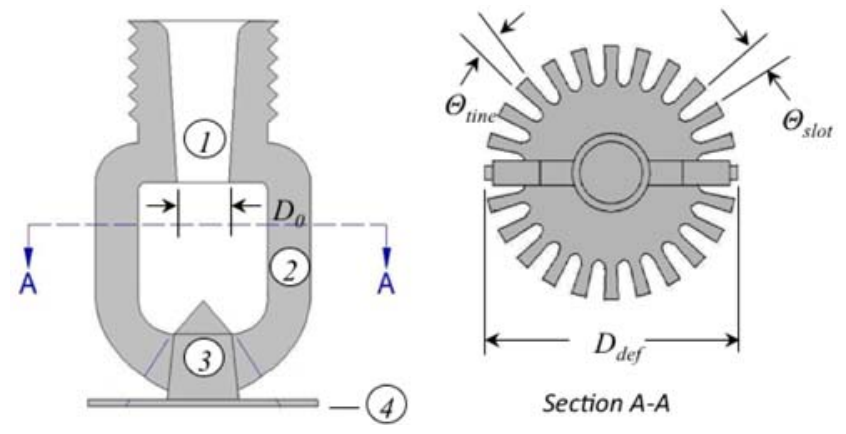

Fig.1. Anatomy of a sprinkler: (1) inlet, (2) frame Arm, (3) boss, (4) deflector.

Despite diversity in size, shape, and design details, most modern fire sprinklers use the same fundamental method of spray generation. Water is initially forced through an orifice to produce a continuous water jet. This jet then impinges onto a deflector to form thin sheets of water. These thin unstable sheets easily disintegrate into drops. A number of basic research studies have reported measurements and theories describing the breakup of these thin liquid sheets. This body of work provides insight into spray generation details for fire sprinklers. A few fundamental atomization studies relevant to sprinklers are presented in the following. Dombrowski and Hooper developed mathematical equations to describe the sinuous and dilatational break-up modes on thin sheets created with fan-spray nozzles [27]. They also extended these equations to predict characteristic wavelengths and drop sizes in each break-up mode. These analytical results compared favorably to some limited experimental data obtained by using high speed flash (i.e., short exposure time) photography. Since these break-up modes have been observed in sprinkler spray generation $[25,26,28]$ for a wide range of operating conditions, Dombrowski and Hooper's analysis among others provides insight for sprinkler sprays, despite differences in nozzle configuration. Correspondingly, Huang utilized a high-speed motion photographic technique to study the break-up mechanisms of liquid sheets formed by the impingement of two co-axial jets [29]. He reported three break-up regimes (depending on We) and their trends by plotting the ratios of break-up radii over nozzle radius against We. In the highest We regime (most relevant to sprinklers) in the range from 2000 to 30000, large sinuous disturbances are observed on the sheet. Ring-like ligaments appear in this regime, and drops are formed when these ligaments disintegrate. The sheet break-up distance was shown to follow the scaling law $r_{b u, s h} / D_{o} \sim W e^{-1 / 3}$. More recently, Clanet and Villermaux conducted a series of experiments to study the formation and disintegration of smooth and flapping liquid sheets, generated by impinging a jet onto a flat deflector [30]. They found break-up distance trends similar to those reported by Huang despite differences in experimental configuration. The arithmetic drop diameter was also examined. Clanet and Villermaux found that this mean diameter can be described by $d / D_{o} \sim\left(\rho_{a} / \rho_{l}\right)^{-2 / 3} W e^{-1}$ over a relatively narrow and small We (1000 to 2000). Unfortunately, little data is available for the high We regime relevant to fire sprinkler operating conditions $\left(3 \times 10^{3}\right.$ to $\left.2 \times 10^{5}\right)$ [25]. A series of detailed experiments have been performed at the University of Maryland to quantify the discharge characteristics from laboratory configurations (i.e. a solid deflectors similar to Clanet and Villermaux) and actual fire sprinkler configurations in this high We regime [24-26].

The sinuous disturbances observed by Dombrowski and Hooper; Huang; and Clanet and Villermaux are captured beautifully in a shadowgraphy image of sheet breakup presented in Fig. 2a. The thin sheet is traveling from top to bottom. The interaction between the liquid sheet and the induced flow are revealed through a tracer introduced in the surrounding air. Infinitesimal disturbances intrinsic to the flow grow exponentially on the sheet, eventually forming large aerodynamic waves that ultimately fragment the sheet. An illustration of similar wave growth and breakup processes responsible for spray formation in fire sprinklers is provided in Fig. $2 \mathrm{~b}$. 


\section{Measurements}

To facilitate measurement and analysis of fire sprinkler sprays, it is useful to divide the process by which water is delivered to protected surfaces into the discrete steps described as injection, breakup, initiation, dispersion, and delivery. The injection condition determined by the injector geometry, injection pressure, and fluid properties governs the topology of the unstable streams, while the flow and fluid properties govern the stream breakup behavior and the resulting initial spray properties. The interaction of the initial spray with the surrounding gas flow governs dispersion and ultimately determines the volume flux delivered to protected surfaces. A variety of measurement approaches were applied to fire sprinklers at each step of the spray delivery process to unravel the sprinkler spray. Qualitative injection information (i.e sheet topology before breakup) was obtained using advanced imaging techniques [21,22] while global information about the sheet structure was obtained using a simple mechanical measurement approach [22]. These approaches revealed how the jet formed in the orifice is transformed into thin unstable streams. Direct photography and shadowgraphy techniques were used to image these streams [21-23]. Breakup locations were easily obtained from the calibrated images, which were useful for comparison with stability theory and atomization model development.

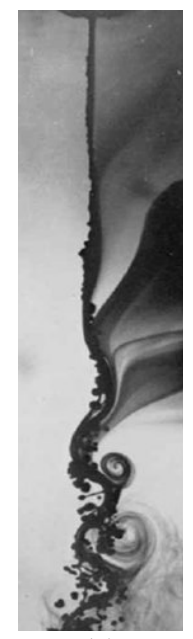

(a)

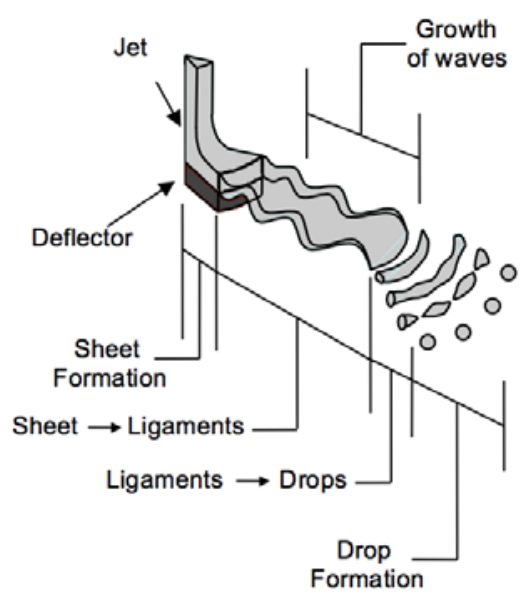

(b)

Fig. 2. (a) Wave growth on a liquid sheet [31]; (b) Spray formation [24].

Drop sizes were measured at a number of stations to map out drop numbers, sizes, and velocities on a hemisphere very close to the injector using a combined shadowgraphy / particle tracking velocimetry (PTV) technique, yielding complete characterization of the initial spray (just after breakup is completed) [26]. Volume flux measurements were also performed one meter below the nozzle to characterize dispersion and wetting from the fire sprinkler sprays [26]. A maximum inviscid reach was defined, $R=U(2 h / g)^{1 / 2}$, where $h$ is the measurement elevation below the nozzle, $g$ is the gravitational constant. This length scale was used to normalize the radial coordinate $r^{\prime}=r / R[16]$. The volume flux was expressed in terms of a linear density, $q^{\prime}=\left(2 q^{\prime \prime} r^{\prime}\right) /\left(Q / \pi R^{2}\right)$, where $q^{\prime \prime}$ is the area volume density and $Q$ is the nozzle flow rate. These measurements combined with analysis have provided novel results and unique insight into fire sprinkler discharge characteristics. This analysis has formed the basis of atomization models, scaling laws, spray initiation approaches, and dispersion modeling ideas.

\section{RESULTS}

\section{Spray Topology}

The sprinkler deflector transforms the jet into a complex unstable sheet structure. For pendent sprinklers, this three-dimensional sheet is approximated by discrete streams formed by flow deflected along the tines 
and by flow forced through the slots as illustrated in Fig. 3a. The short-time exposure photograph of the tine stream in Fig. $3 \mathrm{~b}$ and the shadowgraphy image of the slot stream in Fig. $3 \mathrm{c}$ show that the tine and slot streams appear to break up due to the aerodynamic wave growth mechanisms discussed previously. This is an important finding, which partially validates some of the earlier atomization modeling work performed by Di et al. [28]. Although in this earlier study only the tine stream was considered, as the existence of the orthogonal stream had not yet been discovered. Some insight into the spray topology can also be inferred from a mechanical measurement technique first proposed by Blum [21]. In this technique, the sprinkler is placed inside of concentric cylindrical containers so that its deflector is aligned with a splitter plate, which diverts the slot flow into the inner container and the tine flow into the outer container. Adjusting the elevation of the sprinkler with respect to the splitter plate can reveal additional sprinkler flow stream details, namely estimates of the concentrated around the frame arms and the flow deflected upward for ceiling

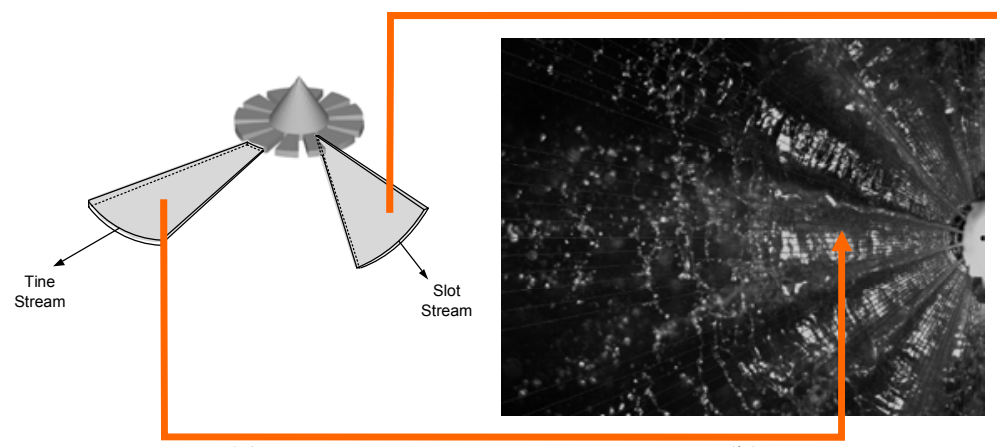

(a) (b)

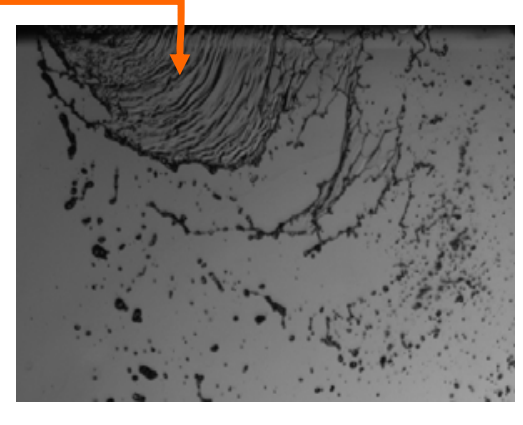

(c)

Fig. 3. Deflector separates the jet into distinct streams; (a) deflector; (b) tine stream photograph (top view); (c) slot stream shadowgraph (side view).

cooling. For example, a Viking VK102 sprinkler having K-factor of $81 \mathrm{~L} / \mathrm{min} / \mathrm{bar}^{1 / 2}\left(D_{o}=12 \mathrm{~mm}\right)$ had the following flow split characteristics. Approximately $77 \%$ of the flow was directed through the slots, while $20 \%$ of the flow was directed along the tines. A splash basin in this deflector directs a small amount $(<0.8 \%)$ of flow upward for ceiling cooling. The flow split measurement technique also revealed that between $2.2 \%$ and $3.0 \%$ of the flow was concentrated around the frame arms. This concentrated flow around the frame arms in fact represents a third type of stream generated by fire sprinklers. Unlike the tine and slot streams, no detailed spray characterization measurements have been performed in the vicinity of the frame arms. There is an immediate need for these detailed measurements to obtain a complete view of discharge characteristics from fire sprinklers. It is also important to understand to what extent the frame arm streams affect the tine and slot streams. This data is crucial for advancing atomization modeling ideas to account for the circumferential volume flux and drop size variations resulting from the flow obstruction caused by the sprinkler frame arms (i.e. the shadowing effect).

\section{Scaling Laws}

Recognizing that the three dimensional topology of the thin sheet results from distinct flow streams (deflected along the tines, forced through the slots, and diverted around the frame arms), it is useful to evaluate discharge characteristics accordingly. Scaling laws have been developed for characteristic sheet breakup locations and drop sizes in terms of physically meaningful parameters constructed from the sprinkler injection details including sprinkler geometry, ambient conditions, and liquid flow properties [22]. These scaling laws reveal how the sprinkler geometry modifies the important physical processes of spray generation responsible for the overall discharge characteristics of the sprinkler. Specifically, dimensionless relationships are developed for each stream to describe sheet formation, sheet breakup, and drop formation. A detailed development of these scaling laws is provided by Do [22] and Ren [14]. This analysis is summarized in the following.

As the flow travels along the deflector, a boundary layer develops reducing the velocity of the thin film. Although the film thickness decreases as it travels radially outward along the deflector, the viscous 
interaction with the deflector decelerates the sheet resulting in a thicker sheet than that expected from inviscid flow theory. The sheet thickening factor at the edge of the deflector, $\beta=U_{o} / U_{s h}$, assumed to be the same for the tine and slot sheets as a first approximation, can be expressed as

$$
\beta=1+0.0564 \frac{R e^{-1 / 5}}{\gamma_{t}}\left(\frac{D_{d}}{D_{o}}\right)^{9 / 5}
$$

where $R e=\rho_{l} U D_{o} / \mu$ is the jet Reynolds number, $\mu$ is the liquid viscosity, $\gamma_{t}$ is the tine flow split factor, and $D_{d}$ is the deflector diameter. The quantity describing the flow distribution between the streams deserves further discussion. It describes the ratio of the stream-wise flow split to the stream-wise geometric area split so that $\gamma_{t}=1$ represents a deflector that geometrically balances the flow. However, for example, when $\gamma_{t}<1$ flow in the tine streams is reduced and a greater share of flow is directed through the void spaces in the deflector resulting in a thinner tine stream. The tine flow split factor can be determined from $\gamma_{t}=\left(Q_{t} / Q_{T}\right) /\left(N_{t} \theta_{t} / 2 \pi\right)$ where the tine flow rate over the total flow rate is based on flow split measurements, $N_{t}$ is the number of tines of the sprinkler, and $\theta_{t}$ is the tine angle. Similarly, the space flow split factor can be determined from $\left.\gamma_{s}=Q_{s} / Q_{T}\right) /\left(N_{t} \alpha / 2 \pi\right)$, where $N_{s}$ is the number of spaces of the sprinkler and $\alpha$ is the angle of the space sheet. This angle can be estimated using the boss angle [22]. The sheet thickening and flow split factors, $\beta$ and $\gamma_{t, s}$ are critically important because they affect the sheet thickness and velocity, which have leading order effects on the breakup process.

After leaving the deflector, the sheet thins as it moves radially outward as illustrated in Fig. 4a. Since the mass of the flow is conserved, the thickness of these sheets can be related to the radial location, sheet geometry, and the flow rate of the nozzle. The sheet thickness for the tine streams and slot streams in terms of these quantities is given by

$T_{t, s} / D_{o}=(1 / 8)\left(\beta \gamma_{t, s}\right)\left(r / D_{o}\right)^{-1}$.

Sinuous wave dispersion equations describing the instability responsible for sheet breakup and spray formation have been applied to the tine stream [24] and later to the slot stream [22]. Based on this wave dispersion theory, the growth of the sinuous wave on a thin inviscid sheet can be described by

$\frac{\partial f}{\partial r}=\left(\frac{4 \rho_{a}^{2} U_{s h}^{2} r}{\rho_{l} \sigma D_{o}^{2} \gamma_{t, s} \beta^{3}}\right)^{1 / 2}$,

where $f$ is the dimensionless wave amplitude and $\partial f / \partial r$ is the dimensionless wave growth rate along the sheet. This wave growth rate depends on the injection properties, the location along the sheet, and the sprinkler geometry. It should be noted that although the sheet thickness does not appear explicitly in the wave growth equation (Eq. 4), the importance of the thickness can be appreciated by recognizing that $T_{t, s}$ is a strong function of $r$ and the sprinkler geometry (i.e. $D_{o}, \beta$, and $\gamma_{t, s}$ ) according to Eq. 5. Integrating Eq. 4 , where $r$ varies from $r_{d}$ to the break-up distance, $r_{b u}$, and $f$ varies from near zero to some critical dimensionless sheet break-up wave amplitude $f_{o}$, yields an expression for the break-up location of the tine (or slot) stream. After considerable rearrangement and assuming that the breakup distance is large with respect to the deflector diameter, the scaling for the sheet breakup can be expressed as

$\left(r_{b u}\right)_{t, s} / D_{o} \sim\left(X_{s h}\right)_{t, s}^{-1 / 3}=\left[\left(\rho_{a} / \rho_{l}\right)\left(f_{o}\right)^{-2}\left(W e / \beta^{3} \gamma_{t, s}\right)\right]^{-1 / 3}$,

where $\left(X_{s h}\right)_{t, s}$ is the sheet breakup parameter for the tine (or slot) stream. The breakup parameter consists of a Weber number modified by nozzle factors affecting the viscous interaction with the deflector and the flow split ( We / $\left.\beta^{3} \gamma_{t, s}\right)$, and factors describing the density ratio $\left(\rho_{a} / \rho_{l}\right)$ and sheet stability $\left(f_{o}\right)$. The sheet 
break-up parameter, $\left(X_{s h}\right)_{t, s}$, integrates nozzle geometry and injection conditions into a single scaling parameter based on wave dispersion theory for evaluation of sprinkler atomization measurements and models.

After the sheet breaks up, the liquid fragments continue to move radially outward forming ring-like ligaments. The volume of a ligament, right after disintegrating from the sheet, can be estimated based on the assumption that the sheet breaks up into a fragment formed from half of the critical wavelength, $\left(\lambda_{\text {crit }}\right)_{t, s}$, with volume

$V_{\text {lig }} \approx(\pi)\left(r_{b u}\right)_{t, s}\left(\lambda_{\text {crit }}\right)_{t, s}\left(T_{b u}\right)_{t, s}$.

It can be shown that the fastest growing or critical wave is selected by the liquid sheet flow and fluid properties so that

$\left(\lambda_{\text {crit }}\right)_{t, s} / D_{o} \approx(4 \pi)\left[\left(\rho_{a} / \rho_{l}\right)\left(W e / \beta^{2}\right)\right]^{-1}$.

By assuming contraction into cylindrical shapes, the ligament diameter can be obtained from Eq. 8 after substituting for $\left(T_{b u}\right)_{t, s}$ from Eq. 5, and $\left(\lambda_{c r i t}\right)_{t, s}$ from Eq. 9, yielding

$d_{l i g} / D_{o}=\left[\left(\rho_{a} / \rho_{l}\right)\left(\left(r_{b u}\right)_{t, s} / D_{o}\right)\left(W e / \beta^{3} \gamma_{t, s}\right)\right]^{-1 / 2}$.

It can be shown that the drop sizes resulting from ligament breakup are directly proportional to the ligament diameter so that substitution of the scaling law for $\left(r_{b u}\right)_{t, s} / D_{o}$ from Eq. 5 into Eq. 8 results in the drop size scaling law

$\left(d_{v 50}\right)_{t, s} / D_{o} \sim\left(X_{d}\right)^{-1 / 3}=\left[\left(\rho_{a} / \rho_{l}\right)^{1 / 2}\left(f_{o}\right)_{t, s}\left(W e / \beta^{3} \gamma_{t, s}\right)\right]^{-1 / 3}$,

where $\left(X_{d}\right)_{t, s}$ is the drop size scaling parameter, which also consists of a Weber number modified by nozzle factors similar to the sheet scaling parameter $\left(X_{s h}\right)_{t, s}$.

The overall breakup and drop size data are evaluated in terms of these scaling laws in order to understand the effect of sprinkler geometry and injection conditions on the discharge characteristics. A number of sprinkler geometries were characterized including geometries with slots and tines (standard nozzles) and solid deflector geometries (basis nozzles). Nozzle details are provided by Do [22]. Correlated sheet data are shown in Fig. 4. Depending on the injector geometry and injection conditions, the breakup radius can range from approximately 10 to 50 orifice diameters. The ideal basis nozzles with solid disc deflectors breakup much later than the more realistic standard nozzles with the slot streams breaking up earliest. Figure 4a shows that increasing We (i.e. the relative importance of inertial forces to surface tension forces) by increasing either injection velocity or injector diameter facilitates breakup, as expected, resulting in smaller breakup distances. Figure $4 \mathrm{~b}$ clearly demonstrates the power of the scaling laws by incorporating not only the We, but also flow split and sheet thickening factors related to deflector geometry, air to liquid density ratios describing the injection environment, and the critical dimensionless wave amplitude related to the sprinkler geometry. The values for $\beta$ tend to increase with $D_{o}$, while values for $f_{o}$ increase with $D_{o}$ for the basis nozzles, while remaining relatively small and unchanged for the standard nozzles. The complex behavior of these parameters combined with the scaling law correlated the breakup data well. The drop size behavior is more complex. Figure 5a shows that the characteristic drop sizes range anywhere from approximately $5 \%$ to $25 \%$ of the orifice diameter, depending on the injection condition and the sprinkler geometry. For a given We, the flat deflector basis nozzles tend to have the largest drop sizes (normalized by $D_{o}$ ) especially for the smallest nozzles. The drops generated from the tine stream tended to have larger drop size than those created by the slot stream for a given $W e$, but it should be noted that this behavior may change with modifications to the sprinkler geometry and associated $\beta, \gamma_{t, s}$, and $f_{o}$. The standard nozzles 
demonstrated an approximate (-1/3) power law decay with We, although the constant of proportionality varies based upon the injection geometry. However, the We scaling was less clear for the basis nozzles.

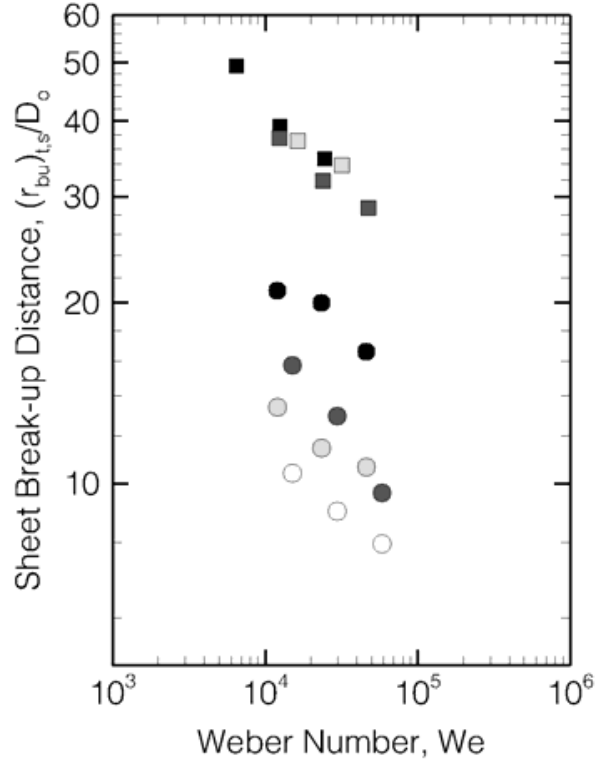

(a)

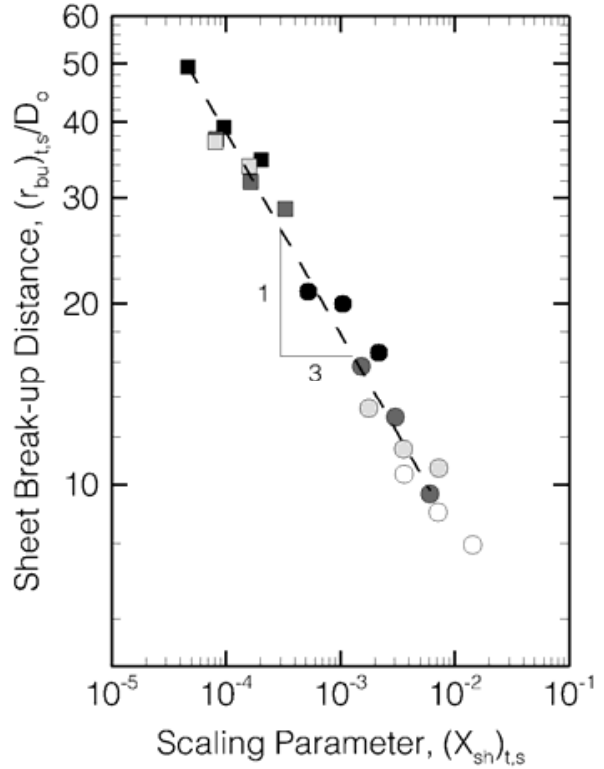

(b)

Fig. 4. Sheet break-up distances: (a) Weber number dependence; (b) scaling parameter dependence. standard nozzles: $\bullet D_{o}=6.2 \mathrm{~mm}$ - tine, $\bullet D_{o}=11.0 \mathrm{~mm}$ - tine, $\odot D_{o}=6.2 \mathrm{~mm}$ - slot, $\bigcirc D_{o}=11.0 \mathrm{~mm}$ slot; basis nozzles: $D_{o}=3.2 \mathrm{~mm}, \square D_{o}=6.2 \mathrm{~mm}, \square D_{o}=9.5 \mathrm{~mm}$

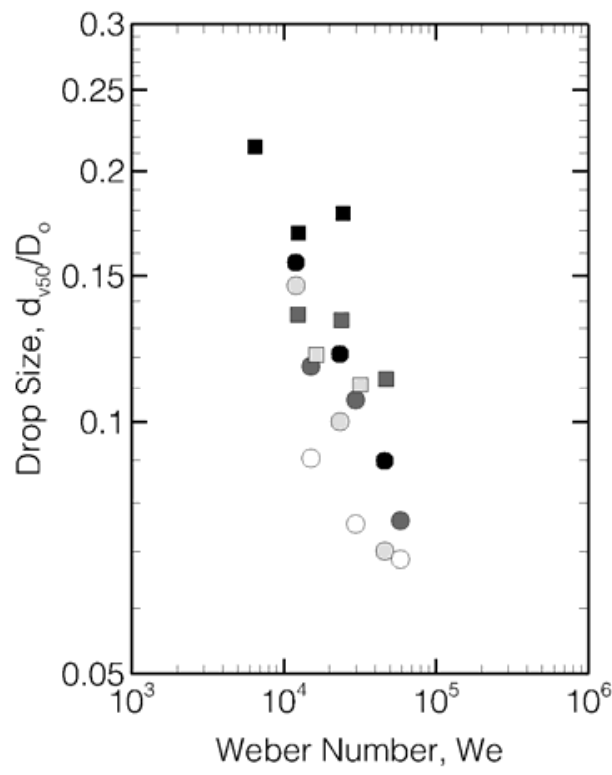

(a)

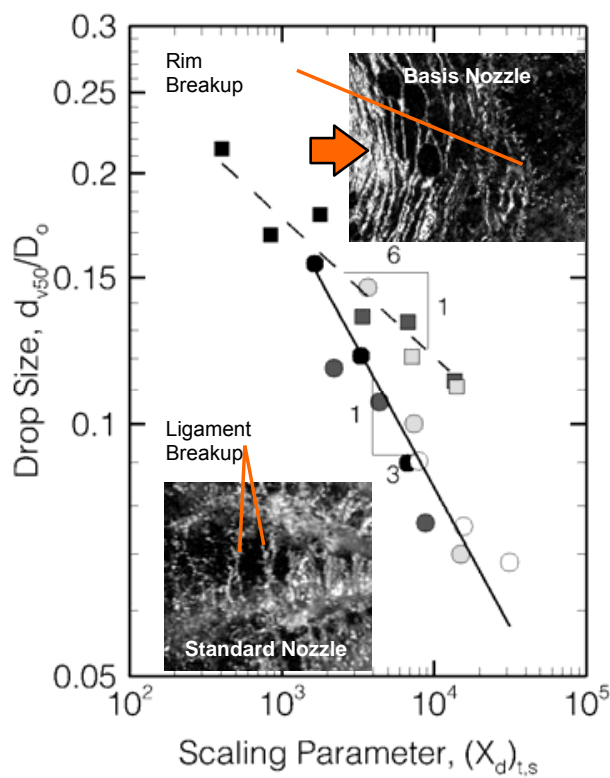

(b)

Fig. 5. Characteristic drop sizes: (a) Weber number dependence; (b) scaling parameter dependence. standard nozzles: $\bullet D_{o}=6.2 \mathrm{~mm}$ - tine, $\bullet D_{o}=11.0 \mathrm{~mm}$ - tine, $O D_{o}=6.2 \mathrm{~mm}$ - slot, $\bigcirc D_{o}=11.0 \mathrm{~mm}$ slot; basis nozzles: $-D_{o}=3.2 \mathrm{~mm}, \square D_{o}=6.2 \mathrm{~mm}, \square D_{o}=9.5 \mathrm{~mm}$ 
Figure $5 \mathrm{~b}$ shows that after the geometry effects $f_{o}, \gamma_{t, s}$, and $\beta$ are included, two scaling behaviors become apparent. The basis nozzles show a more clear trend with the droplet scaling parameter $\left(X_{d}\right)_{t, s}$, having a relatively slow decay $(-1 / 6)$, while the drops from the tine and slot streams of the standard nozzles tend to follow a $(-1 / 3)$ power law behavior with $\left(X_{d}\right)_{t, s}$. The difference in scaling laws can be explained through observations in sheet breakup behavior. For the basis nozzles, the drops appear to form directly at the edge or 'rim' of the sheet as shown in the image provided in Fig 5b. Alternatively, the standard nozzles show that the sheet breaks up into ligaments, which then disintegrate into drops.

The drop size scaling law provided in Eq. 9 was based on this multi-step process and performs well when these conditions are realized. It is therefore no surprise that the drop size scaling law is not valid for the basis nozzle, where the drops appear to be formed directly from the edge or 'rim' of the sheet. However, it is remarkable that the $\left(X_{d}\right)_{t, s}$ parameter correlates the data under the 'rim' drop formation mechanism, albeit, with a power law that differs from the theory.

\section{Detailed Distributions}

The scaling laws provide useful overall spray properties. However, the three dimensional nature of the spray is clearly observed in the sheet breakup images provided in Fig. 3. These inherent multidimensional characteristics of the spray present a critical design challenge for achieving adequate spray distribution uniformity required for fire sprinklers certification.

The multi-dimensional behavior of the spray is further complicated by its stochastic nature. Strictly speaking, unique probability distributions are required at every location within the spray. A spray initiation framework has been developed to address this multidimensional stochastic complexity [26]. Following previous researchers $[19,35]$, the sprinkler spray is established on an initiation sphere. From measurements such as those provided in Fig. 3, sheet breakup and the associated spray formation is usually completed by $r=0.35 \mathrm{~m}$ for most sprinklers. At this radial location, the stochastic spray can be completely described by the azimuthal angle $(\psi)$, the elevation angle $(\theta)$, the volume fraction (or associated number density), the drop size $(d)$, and the drop velocity $(u)$ according to the following equation:

$$
\int_{\theta} \int_{\psi} \int_{u} \int_{d} f_{V}(\theta, \psi, u, d) d \theta \cdot d \psi \cdot d u \cdot d d=1
$$

where $f_{V}$ is a volume based probability density function based on the initial location, drop size, and velocity. Detailed spatially resolved measurements of the initial spray have been conducted to quantify the multi-dimensional spray characteristics. These measurements provided sufficient spray data to map out a spherical shell at azimuthal

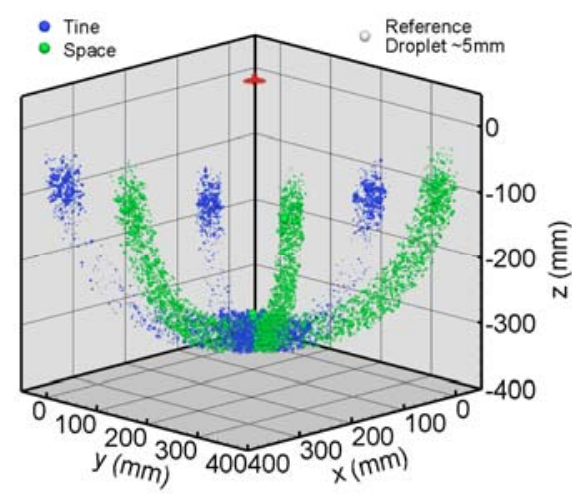

(a)

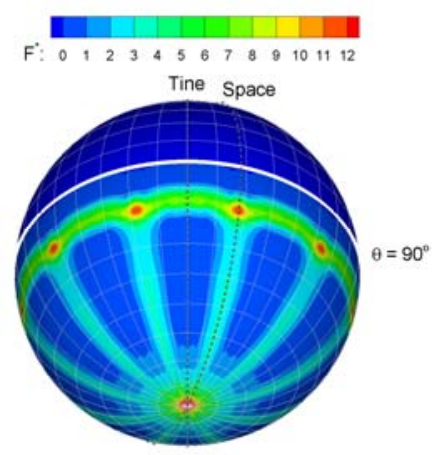

(b)

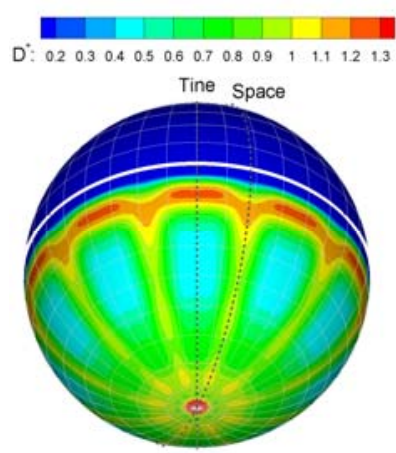

(c)

Fig. 6. Spray characteristics on the initiation sphere $(r=0.35 \mathrm{~m})$ [26]; (a) drop size measurements; (b) flux normalized by average flux; (c) local $d_{v 50}$ normalized by overall $d_{v 50}$. 
angles aligned with the sprinkler tines and slots as shown in Fig. 6a. Only spray from the tine and slot streams are provided in the figure; however, there is an ongoing effort to characterize the spray near the frame arms to provide a truly complete description of the initial spray. Nearly a million drops are measured to produce the volume probability distribution function described in Eq. 10 accounting for the drops in the initial spray. Drops can be defined according to this distribution function to generate a stochastic description of the initial spray. The measurements, while extensive, only account for azimuthal angles aligned with the tine and the slot. Furthermore, the distributions provide no insight into physically meaningful spray quantities such as the spray angle or characteristic drop size. In other words, the distributions, although descriptive, are somewhat cryptic. To address these issues, a series of basis function were defined to provide continuous distributions on the initial sphere. These basis functions are constructed using an array of physically significant coefficients. The coefficients provide tangible and convenient spray characteristics, while combining with basis functions to describe the initial spray with unprecedented detail. Basis functions are used to describe the spatial variation $(\theta$ and $\psi$ ) of the drop characteristics required to define the spray. Basis functions are also used to describe the local drop size distributions.

\section{Velocity}

A very simple approach was used to describe the drop velocity. Based on analysis, the drop velocity vector is aligned with the drop position vector having origin at the center of the deflector. At the initiation sphere, the drops follow rays emanating from this origin, requiring only specification of the velocity magnitude. This velocity magnitude can take on a wide range of velocities depending on the location and drop size; however, it was found that one characteristic velocity magnitude for all drops provides adequate prediction of spray dispersion (discussed in the next section) and greatly simplifies specification of the initial spray [26].

\section{Drop Size}

The modified log-normal Rosin-Rammler drop size distribution proposed by $\mathrm{Yu}$ [13] is used in this framework to describe the local drop size distributions:

$$
C V F(d)=\left\{\begin{array}{lc}
\left.\frac{1}{\sqrt{2 \pi}} \int_{0}^{d \Gamma / 1.15} \frac{\Gamma\left(-\ln \left(d^{\prime} / d_{v 50}\right)\right)^{2}}{2(1.15 / \Gamma)^{2}}\right) d d^{\prime} & \left(d<d_{v 50}\right), \\
1-\exp \left(-0.693\left(d / d_{v 50}\right)^{\Gamma}\right) & \left(d>d_{v 50}\right)
\end{array}\right.
$$

where $C V F(d)$ is the cumulative volume fraction describing the fraction of the spray volume contained in drops having diameter smaller than $d$. The local volume median diameter, $d_{v 50}$, and $\Gamma$ are parameters describing the characteristic drop size and spray width. The stochastic nature of the spray is captured locally with this distribution. In order to account for the changes of the drop size with elevation angle, Legendre polynomials are used to describe the variation of $d_{v 50} / D_{o}$ and $\Gamma$, so that

$$
\begin{aligned}
& f_{d v 50}\left(\theta, \psi_{t, s}\right)=\sum_{n=0}^{\infty} L_{n}\left(d_{v 50}\right) P_{n}\left(\left(\theta-135^{\circ}\right) / 45^{\circ}\right) \\
& f_{\Gamma}\left(\theta, \psi_{t, s}\right)=\sum_{n=0}^{\infty} L_{n}(\Gamma) P_{n}\left(\left(\theta-135^{\circ}\right) / 45^{\circ}\right),
\end{aligned}
$$

where $f_{d v 50, \Gamma}\left(\theta, \psi_{t, s}\right)$ are continuous functions describing $\theta$ variation of spray properties $\left(d_{v 50} / D_{o}\right.$ or $\left.\Gamma\right)$ constructed from Legendre polynomials, $P_{n}$, and Legendre polynomial coefficients, $L_{n}$, determined from spray measurements aligned with the tine or the slot.

In order to account for $\psi$ variation, a Fourier series is defined to describe mixing between the tine and slot streams, so that for example 


$$
\begin{aligned}
& f_{d_{v 50}}(\theta, \psi)=A(\psi) f_{d_{v 50}}\left(\theta, \psi_{t}\right)+(1-A(\psi)) f_{d_{v 50}}\left(\theta, \psi_{s}\right) \\
& A(\psi)=\frac{a_{0}}{2}+\sum_{n=1}^{\infty} a_{n} \cos \frac{n \pi}{T / 2} \psi
\end{aligned},
$$

where the Fourier coefficients are described by the sprinkler deflector geometry as

$$
a_{n}=\frac{2}{T} \int_{-T_{\text {tine }} / 2}^{T_{\text {tin }} / 2} \cos \frac{n \pi}{T / 2} \psi d \psi
$$

A value of $n=2$ was found to provide good agreement between volume flux measurements and predicted fluxes initiated with this value.

\section{Volume Fraction}

Similar to the drop size parameters, $d_{v 50}$ and $\Gamma$, Legendre polynomials are also applied to determine the volume fraction parameter, $f_{V}$, which is a volume probability density function used to determine the initial elevation angle of drops. However, a Gaussian function is added to characterize the high volume concentration near $\theta=0^{\circ}$ associated with the tine stream yielding

$$
f_{V}\left(\theta \mid \psi_{t, s}\right)=\frac{F_{0}}{\sqrt{2 \pi} \sigma} \exp \left(-\frac{\left(\theta-\theta_{0}\right)^{2}}{2 \sigma^{2}}\right)+\sum_{n=0}^{\infty} L_{n}\left(f_{V}\right) P_{n}\left(\left(\theta-135^{\circ}\right) / 45^{\circ}\right),
$$

where $f_{V}\left(\theta \mid \psi_{t, s}\right)$ conditional volume probability distribution aligned with the tine or slot stream having the random variable, $\theta$. The same Fourier series described in Eq. 15 is used to determine $f_{V}(\theta \mid \psi)$ as it varies between tine and slot stream values. Similarly $f_{V}(\psi)$ is also obtained from Eq. 15 and $f_{V}\left(\psi_{t, s}\right)$ from experimental data to determine the initial azimuthal angle of the drops. Figure $6 \mathrm{~b}$ and $\mathrm{c}$ shows plots of the basis functions on the spray initiation sphere capturing the tine and slot stream behavior of a Tyco ESFR sprinkler with K-factor of $202 \mathrm{~L} / \mathrm{min} / \mathrm{bar}^{1 / 2}$. The volume probability density function shows that the spray is concentrated near the equator for the tine and slot flows. However, for azimuthal angles aligned with the slot, the spray is distributed more uniformly over elevation angles. For this ESFR sprinkler the drops created by the tine stream are larger than the drops created by the slot stream. It should be noted that the width of the drop size distribution (not shown) is relatively constant throughout the spray.

The coefficients for the basis functions plotted in Fig. 6 have been compiled, organized and illustrated in Fig. 7a providing physically meaningful parameters to facilitate description and comparison of sprinkler discharge characteristics. Average volume fractions (and corresponding volume probability density) are up to 12 times higher than the average at the peak, while the non-peak volume fractions generated by the tine stream are approximately 2 times higher than that of the slot. Characteristic drop sizes generated from the tine stream are about 1.5 times larger than that generated by the slot stream. While the drop size distribution (not shown) is relatively wide consistent with typical sprinklers having $\Gamma \approx 2.5$. Based on averaged measured values, the velocities are approximated to be $0.7 U$ to initiate the spray.

It is clear that the basis functions provide a useful framework for characterizing the spray in a physically meaningful compact format. However, this framework may also be useful for predicting the initial spray. For example, the scaling laws developed for $d_{v 50}$ could be used to predict $L_{o}\left(d_{v 50}\right)$ using the injection conditions and injector geometry. The Fourier coefficients are also available directly from the injector geometry. It may also be possible to develop scaling laws for other parameters or estimate their values based on experience. In order to explore typical values or ranges of values for these parameters, much more experimental data is needed. It would be useful to create a sprinkler database in the context of the framework, where the coefficients could be easily compared among sprinklers facilitating insight into the spray characteristics relationship with geometry. 


\section{Dispersion}

Dispersion measurements have been made to evaluate the sprinkler spray characterization framework and to visualize the sprinkler dispersion. Flux measurements $1 \mathrm{~m}$ below the deflector were compared with dispersion predictions. The analytical spray was generated in still air and tracked from the initiation location using a Lagrangian particle tracking method and assuming one way coupling between the spray and the surrounding air [26]. The simplifying one-way coupling assumption is expected to be valid in this momentum dominated region of the spray. Comparisons between the predicted and measured flux distribution for the ESFR sprinkler are provided in Fig. 7b. The linear density of volumetric flux shows good agreement between the predicted and measured fluxes $1 \mathrm{~m}$ below the nozzle, revealing not only the accuracy of the compression approach, but also

\section{Spatial Variation (Elevation Angle, $\theta$ )}
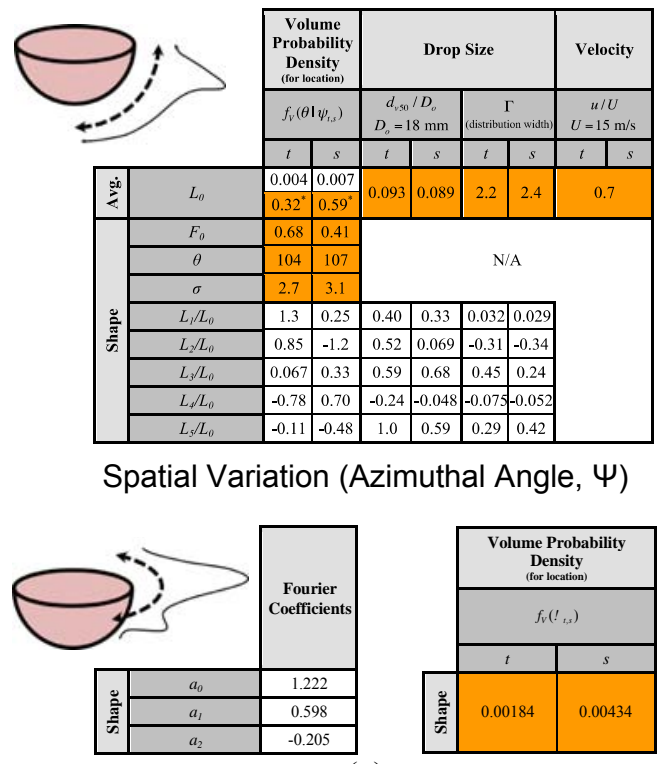

(a)

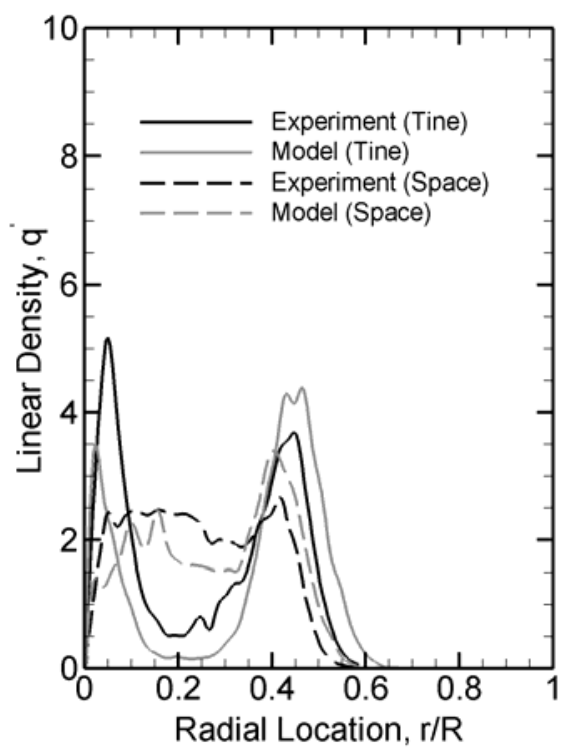

(b)

Fig. 7. ESFR sprinkler data (K-factor: $202 \mathrm{~L} / \mathrm{min} / \mathrm{bar}^{1 / 2}$ ) operating at $1.1 \mathrm{bar}$; (a) description of coefficients for the spray initiation basis functions ; (b) comparison of volume flux distribution $1 \mathrm{~m}$ below sprinkler [26].

the suitability of the simplified air-drop coupling for particle tracking near the nozzle exit. More careful coupling will be required to determine induced flow and to calculate fire plume interaction. Even in the quiescent environment, more detailed coupling will be required in the near-field to account for ceiling effects and in the far-field to account for air entrainment effects. The distinct tine and slot features are clearly observed after dispersion $1 \mathrm{~m}$ below the nozzle. The linear flux aligned with the tine shows a double peak structure with a high density near the axis of the sprinkler and in the outer regions of the spray, while the linear flux aligned with the tine shows a more uniform flux. The agreement achieved between the predictions initiated with the basis functions and the measurements is noteworthy, especially considering the simplifying assumptions that were made for the injection velocity and the one way coupling used for droplet tracking.

\section{SUMMARY}

Characterizing fire suppression sprays, and in particular sprinkler sprays represents a daunting challenge because of their complexity. At the same time, this is a problem with great impact because of the ubiquity of fire sprinklers, their important task, and the need for this information in computationally based analysis tools, which are becoming increasingly popular in the practice of fire protection engineering. To meet this challenge, sophisticated measurement methods have been applied to the sprinkler spray along with classical 
stability theory to understand the process by which the sprays are formed as the jet is transformed into distinct thin sheets by the sprinkler geometry (i.e. the deflector) and ultimately fragmented to generate the spray. Scaling laws were developed from the theory and compared with overall spray measurements with some success. However, the spatial variation of the spray is unwieldy. To bring some order to the stochastic spatially varying spray, a framework of physically significant basis functions was developed to characterize the initial spray. These basis functions are generated from a tractable number of physically meaningful coefficients generated from the experimental data, providing the opportunity to quantitatively evaluate the sprinkler spray at a deeper level of detail. Although, a number of accomplishments have been made toward unraveling the sprinkler spray, there is still quite a jumble to sort through. Understanding the effect of the frame arms on the initial spray and integrating these effects into the previously presented analytical techniques stand as the single biggest immediate task. Now that a framework has been established, a sprinkler database should follow which would aid in understanding how geometrical differences impact the spray details. This framework should also be integrated into popular CFD codes, which would provide a convenient means for high fidelity spray specification. Finally, there is a real need to move away from empiricism and toward the development of a sprinkler atomization model (SAM) that would generate a detailed representation of the initial spray from calculations of the interaction between the liquid injected from the sprinkler orifice, the sprinkler geometry, and the surrounding flow conditions. It is hoped that the challenges and progress in the area of fire sprinkler sprays will inspire new approaches and discoveries for fire sprinklers and other fire suppression devices.

\section{ACKNOWLEDGEMENTS}

This work is supported by FM Global and the National Science Foundation under Award \# 0645063. The author would like to thank the program manager Dr. Bert Yu of FM Global and the IAFSS program committee for their patience and confidence as this manuscript was being prepared.

\section{REFERENCES}

[1] Rasbash, D.J., 1986. The Extinction of Fire with Plain Water: A Review. Fire Safety Science 1: 1145-1163. http://dx.doi.org/10.3801/IAFSS.FSS.1-1145

[2] Grant, G., Brenton, J., and Drysdale, D., (2000). Fire Suppression by Water Sprays, Progress in Energy and Combustion Science, 26: 79-130. http://dx.doi.org/10.1016/S0360-1285(99)00012-X

[3] Yao, C., (1988). The Development of the ESFR Sprinkler System, Fire Safety Journal, 14(1-2): 6573. http://dx.doi.org/10.1016/0379-7112(88)90045-8

[4] Nash, P., and Rasbash, D., "The Use of Water in Fire-Fighting," Fire Research Station, Borehamwood, UK, 1955.

[5] Mawhinney, J. R. and Richardson, J. K., (1997). A Review of Water Mist Fire Suppression Research and Development, Fire Technology, 33(1): 54-90. http://dx.doi.org/10.1023/A:1015322428719

[6] Ndubizu, C.C., Ananth, R., Tatem, P.A., and Motevalli, V., (1998). On Water Mist Fire Suppression Mechanisms in a Diffusion Flame, Fire Safety Journal, 31(3): 243-276. http://dx.doi.org/10.1016/S0379-7112(98)00007-1

[7] Heskestad, G., (2003). Extinction of Gas and Liquid Pool Fires with Water Sprays, Fire Safety Journal, 38(4): 301-317. http://dx.doi.org/10.1016/S0379-7112(02)00085-1

[8] Heskestad, G., (2002). Scaling the Interaction of Water Sprays and Flames, Fire Safety Journal, 37(6): 535-548. http://dx.doi.org/10.1016/S0379-7112(02)00012-7

[9] Yu, H.Z., Zhou, X. and Ditch, B., 2009. Experimental Validation of Froude-modeling-based Physical Scaling of Water Mist Cooling of Enclosure Fires. Fire Safety Science 9: 553-564. http://dx.doi.org/10.3801/IAFSS.FSS.9-553

[10] Dundas, P.H., "The Scaling of Sprinkler Discharge: Prediction of Drop Size," Factory Mutual Research Corporation No. 18792 RC73-T-40, Norwood, MA, 1974.

[11] Heskestad, G., "Proposal for studying Interaction of Water Sprays with Plume in Sprinkler Optimization Program”, Norwood, MA, 1972. 
[12] Schick, R.J., “An engineer's practical guide to drop size”, Spraying Systems Co. Bulletin 459, Wheaton, IL, 1992.

[13] Yu, H.Z., 1986. Investigation of Spray Patterns Of Selected Sprinklers With The FMRC Drop Size Measuring System. Fire Safety Science 1: 1165-1176. http://dx.doi.org/10.3801/IAFSS.FSS.1-1165

[14] Ren, N., "Advances in Characterizing Sprinkler Sprays," PhD Thesis, University of Maryland, College Park, USA, 2010.

[15] Beyler, C., "Effect of Selected Variables on the Distribution of Water from Automatic Sprinklers," NIST NBS GCR 77-105, Gaithersburg, MD 1977, 533 p.

[16] Prahl, J., and Wendt, B., (1988). Discharge Distribution Performance for an Axisymmetric Model of a Fire Sprinkler Head, Fire Safety Journal, 14(1-2):101-111. http://dx.doi.org/10.1016/0379$\underline{7112(88) 90048-3}$

[17] Widmann, J.F., (2001). Phase Doppler interferometry measurements in water sprays produced by residential fire sprinklers, Fire Safety Journal, 36(6): 545-567. http://dx.doi.org/10.1016/S03797112(01)00009-1

[18] Sheppard, D.T., and Gandhi, P.D., "Understanding Sprinkler Sprays: Trajectory Analysis," National Institute of Standards and Technology Report NISTIR 6561, Gaithersburg, MD, 2000.

[19] Sheppard, D.T., "Spray Characteristics of Fire Sprinklers," National Institute of Standards and Technology Report NIST GCR 02-838, Gaithersburg, MD, 2002.

[20] Putorti, A.D., "Simultaneous measurements of drop size and velocity in large-scale sprinkler flows using particle tracking and laser-induced fluorescence," National Institute of Standards and Technology Report NIST GCR 861, Gaithersburg, MD, 2004.

[21] Blum, A., "Discharge Characteristics of Canonical Sprinkler Sprays," MS Thesis, University of Maryland, College Park, USA, 2007.

[22] Do, C., "Stream-wise Discharge Characteristics of Pendant Sprinkler Sprays," MS Thesis, University of Maryland, College Park, USA, 2009.

[23] Ren, N., "Analysis of the Initial Spray from Canonical Fire Suppression Nozzles," MS Thesis, University of Maryland, College Park, USA, 2007.

[24] Ren, N., Blum, A., Zheng, Y., Do, C. and Marshall, A., 2009. Quantifying the Initial Spray from Fire Sprinklers. Fire Safety Science 9: 503-514. http://dx.doi.org/10.3801/IAFSS.FSS.9-503

[25] Ren, N., Blum, A., Do, C. and Marshall, A., (2009). Atomization and dispersion measurements in fire sprinkler sprays, Atomization and Sprays, 19:1125-1136. http://dx.doi.org/10.1615/AtomizSpr.v19.i12.30

[26] Ren, N., Baum, H., and Marshall, A., (2010). A comprehensive methodology for characterizing sprinkler sprays, Proceedings of the Combustion Institute, 33(2): 2547-2554. http://dx.doi.org/0.1016/j.proci.2010.06.10

[27] Dombrowski, N., and Hooper, P., (1962). The effect of ambient density on drop formation in sprays. Chemical Engineering Science, 17(4): 291-305. http://dx.doi.org/10.1016/0009-2509(62)85008-8

[28] Wu, D., Guillemin, D., and Marshall, A. (2007). A modeling basis for predicting the initial sprinkler spray. Fire Safety Journal, 42(4): 283-294. http://dx.doi.org/10.1016/j.firesaf.2006.11.007

[29] Huang, J.C.P., (1970). Break-up of Axisymmetric Liquid Sheets, Journal of Fluid Mechanics, 43(2): 305-319. http://dx.doi.org/10.1017/S0022112070002392

[30] Villermaux, E., and Clanet, C., (2002). Life of a flapping liquid sheet. Journal of Fluid Mechanics, 462(1): 341-363. http://dx.doi.org/10.1017/S0022112002008376

[31] Crapper, G.D., Dombrowski, N. and Pyott, G.A.D., (1975). Large-Amplitude Kelvin-Helmholtz Waves on Thin Liquid Sheets, Proceedings of the Royal Society of London, 342(1629): 209-224. http://dx.doi.org/10.1098/rspa.1975.0021 